\title{
Comunicación y procesos de socialización empresarial
}

\author{
Rocío del Pilar HUERTA ÁLVAREZ
}

Los aportes de diferentes especialistas al análisis de la comunicación empresarial posibilitan, al menos a cierto nivel teórico, la comprensión cabal de la importancia e influencia de este proceso para la consecución de determinados objetivos. Sin embargo, en la práctica observamos que se presentan resistencias e incluso podriamos afirmar, basados en investigaciones previas y sin temor a equivocarnos, que existen prejuicios respecto de las potencialidades y beneficios que ofrece la comunicación como medio de transmisión de mensajes, actitudes e imágenes, para el establecimiento de una cultura institucional integral.

Las razones de este "escepticismo" son diversas. Entre ellas nos limitaremos a señalar sólo dos, a saber, la que se refiere a la demora en obtener resultados tangibles, expresados en términos de rentabilidad económica, y la otra que se funda en el excesivo esfuerzo que se requiere en tiempo y personal para impulsar estas actividades de comunicación empresarial.

Como se observa con frecuencia, el quehacer directivo empresarial se orienta a atender lo inmediato, es decir a lo fundamental para sobrevivir, enfrentando elementos entrópicos como la competencia (a nivel externo), o una huelga del sindicato de trabajadores (a nivel interno). De ningún modo afirmamos que lo anterior esté mal, pues entendemos las presiones y demandas que un líder formal soporta en la actualidad. Sin embargo, consideramos que su función directiva se vería menos recargada si ésta contara con la transmisión efectiva de mensajes que tradujeran una filosofia empresarial integral. 
1. Myers, Gail E. [y] Michelle Tolela Myers.

Administración mediante la comunicación: Un enfoque organizacional. México: Editorial McGraw Hill, 1983, p. 23.
Observamos que en la cotidianidad de la vida institucional se presentan circunstancias en las que predomina la búsqueda de un alto nivel de eficiencia, descuidando o no prestando atención a los mensajes de tipo verbal y no verbal. Esta actitud, frecuentemente no intencional, puede provocar consecuencias negativas observables en el nivel motivacional de los empleados, o en los grados de identificación y pertenencia de éstos respecto de la empresa, lo que podría evitarse e incluso se les podría motivar en sentido favorable, en beneficio de la organización.

Detengámonos por un momento en los procesos de reclutamiento, selección y socialización del personal, prestando mayor atención a este último, por ser al que le dedicaré más espacio en este artículo. Cabe indicar la interdependencia que existe entre estos procesos, por lo que se encuentran unidos inextricablemente en el análisis de la vida organizacional.

El público interno está compuesto por el personal activo de la entidad, entre cuyos integrantes se da un contacto cercano e interdependiente con el fin de lograr los objetivos productivos. El denominado "recurso humano" es el que, según Tolela Myers, "le otorga vida a la organización" 1 , y no sólo cuenta con conocimientos sino también con un nivel motivacional que, en determinadas circunstancias, influye en la prestación óptima del servicio ofrecido.

Los sistemas organizacionales, dinámicos y cambiantes, requieren de un flujo permanente y recurrente de recursos materiales y humanos. De aqui se deriva que el indispensable proceso de captación y selección de personal adquiera niveles de rigurosidad orientados a contratar a aquellas personas que coincidan con el perfil profesional requerido.

El reclutamiento es una actividad que realizan las instituciones después de analizar exhaustivamente sus necesidades, para captar nuevos trabajadores. Los autores William B. Werther y Keith Davis lo definen como:

\footnotetext{
"El proceso de encontrar y atraer solicitantes adecuados para los empleos. Este se inicia cuando se buscan nuevos candidatos y concluye cuando se presentan sus solicitudes. El resultado es un conjunto de buscadores de empleos entre los que se escoge a los nuevos miembros del personal" 2 .
}

Generalmente este proceso es responsabilidad del área de recursos humanos, la que tiene por misión identificar 
cada cierto tiempo los requerimientos de personal mediante las técnicas y métodos apropiados para ello.

El objetivo del reclutamiento es buscar y atraer personas que tengan la capacidad de cubrir las plazas disponibles, para lo cual resulta adecuada la política de considerar que las vacantes sean cubiertas con el público interno y luego recurrir a fuentes externas. El propósito de la comunicación en este proceso se orienta hacia la persuasión de los "empleados potenciales", a quienes se les informa de modo objetivo y realista acerca de las ventajas y oportunidades que ofrece la empresa. Se debe evitar el exceso de vanagloria al realizar la convocatoria, pues en lugar de obtener una imagen favorable, se fomentará una perjudicial y contraproducente. También es fundamental que la comunicación sea efectiva, es decir, que se asegure la comprensión cabal de los mensajes, para lo cual la elección de los medios debe ser el resultado de un análisis realista de las ventajas y limitaciones que ofrecen.

Luego de esta etapa se continúa con la selección de candidatos, realizando el estudio de las habilidades y capacidades de los postulantes, con el fin de decidir quiénes poseen las cualidades deseadas según el puesto de trabajo. La selección es la actividad realizada para atraer a un conjunto de personas con determinadas características y elegir a las de mayor competencia para desempeñar los cargos previamente definidos.

Es evidente que los individuos y las organizaciones se atraen y se seleccionan mutuamente sobre la base de sus motivaciones, necesidades y expectativas, produciéndose entre ambos un ajuste mutuo con miras al desarrollo recíproco. Estas transacciones son fruto de la percepción acerca de las oportunidades que se ofrecen y son difundidas a través de una estrategia comunicacional, así como de su constatación en la realidad al obtenerse apreciaciones de otros individuos relacionados con la empresa. Es obvio que cuanto más satisfecho esté un trabajador, más potente es la fuerza que se ejerce sobre él para que permanezca en el trabajo, y son menores las probabilidades de que lo abandone voluntariamente. Diversos estudios han comprobado que el nivel de desempeño está en relación directa con la cantidad de motivación: "Cuanto más motivado esté el empleado para desempeñar su labor, más efectiva será su capacidad"3. Por consiguiente, es primordial que se realice una adecuada selección, pues a través de ella se elige y se incorpora a quienes formarán parte de la entidad. Dada la trascendencia e influencia de esta etapa en el desarrollo institucional, ella

\author{
3. Arias Galicia, \\ Fernando. "La \\ motivación", en \\ Rodil Urrego, F. y F. \\ Mendoza Trejo \\ (editores), Conceptos \\ fundamentales sobre \\ la organización. \\ México: Editorial \\ Trillas S.A., 1979, p. \\ 113.
}


4. Para mayor información sobre el tema acudir a la tesis: "La comunicación organizacional, los programas de inducción de personal y su importancia en la dinámica de la organización", presentada por Rocio Huerta Álvarez. Lima: Universidad de Lima, Facultad de Ciencias de la Comunicación, 1991. debe sustentarse en un plan integral claramente establecido y conocido por todos los integrantes del sistema.

Las implicancias e importancia de las etapas explicadas antes son ampliamente conocidas. Cabe indicar, en cambio, que respecto de los procesos de socialización del personal de reciente ingreso no hay una percepción definida, quizás porque se presupone que aquello se realiza de manera "mecánica". A continuación revisaremos algunos puntos específicos.

\section{CARACTERÍSTICAS DEL GRUPO QUE INGRESA A UNA EMPRESA}

Por lo general, los empleados de reciente contratación necesitan de cierta orientación y adiestramiento antes de que puedan desempeñar plenamente sus obligaciones laborales. Es conveniente introducirlos en el ambiente en el que se van a desenvolver entregándoles la información respectiva, de tal manera que se familiaricen con la cultura organizacional existente. Así, al complementarse las capacidades de los antiguos y nuevos trabajadores se produce un equilibrio homeostático entre lo que exige el empleo y lo que pueden hacer los recién contratados.

Podría reconocerse que quienes ingresan a trabajar tienen un alto grado motivacional y expectativas favorables hacia la entidad que los contrató, por lo que es de vital importancia ubicar a estas personas según sus aptitudes, intereses y capacidades. Es un hecho, también, que el individuo que ingresa a una institución se enfrenta por lo general con una atmósfera desconocida, en la que encuentran a personas extrañas y un puesto de trabajo que no domina en su integridad. Esto hace que el trabajador adopte una actitud de inseguridad, puesto que aún no ha internalizado los conceptos que forman la totalidad de la organización a la que se ha incorporado. Este periodo de adaptación será particularmente dificil si la entidad no ha contemplado la situación, no obstante que de la respuesta del nuevo empleado dependerá su futura adecuación en la empresa.

\section{LA ACOGIDA DEL PERSONAL}

Es entendida como una técnica de formación que debe incluirse en el programa inductivo de la entidad ${ }^{4}$, coincidente con las normas y criterios existentes. Su contenido es sobre todo informativo y se orienta a que el nuevo empleado 
conozca la organización en su totalidad, se integre a ella y esté motivado a colaborar de manera espontánea.

\section{PROCESO DE SOCIALIZACIÓN DEL PERSONAL DE RECIENTE INGRESO}

Este proceso resulta importante porque influye decisivamente en las actitudes futuras de los trabajadores con respecto a la empresa, al grupo de trabajo y al puesto que ocupan. El ingreso constituye la etapa de mayor receptividad de instrucciones y normas de conducta, a la par que en ella se promueve la afiliación al grupo laboral y la adquisición de hábitos aceptados en el entorno corporativo. En razón de lo anterior, las estrategias de comunicación deben ser diseñadas por profesionales en comunicación social, quienes estudiarán a su público objetivo considerando sus necesidades, motivaciones, características y particularidades concretas. No olvidemos que la corporación tiene una responsabilidad social no sólo hacia sus clientes, sino también hacia sus trabajadores, siendo fundamental la comprensión e internalización de la filosofia, cultura y clima organizativo, ofreciéndoles asesoría y ayuda para su integración y adaptación.

Por desgracia, aún son muchas las instituciones que no son conscientes de la real importancia que tiene una politica comunicacional integral. Se observa la falta de compromiso e interés por parte de los directivos en compartir información con otros miembros de la empresa, ignorando que estos vacíos son sumamente perjudiciales.

\section{RESPONSABILIDAD DE LOS PROGRAMAS DE SOCIALIZACIÓN}

Sería absurdo otorgarle esta responsabilidad a una sola área de la empresa, pues conforme a la visión sistémica de las corporaciones, éstas deben entenderse como un sistema, es decir, como la "combinación de unidades o partes existentes en un entorno, siendo la interdependencia una característica fundamental del mismo" 5 . No podemos olvidar que este proceso involucra a diferentes departamentos: Administración de Personal, Seguridad Industrial, Bienestar Social y, por supuesto, Comunicaciones, sin olvidar la participación del área específica en la que se insertará el nuevo trabajador. Todos ellos tienen una responsabilidad en las distintas etapas y, sobre todo, el compromiso irrenunciable de lograr la

5. Paredes Guzmán, Carlos. "Concepto de sistemas". Lima: Universidad de Lima, Facultad de Ciencias de la Comunicación, 1991. 
adaptación integral beneficiosa tanto para el empleado como para la empresa. No hay que olvidar, sin embargo, que a veces el celo profesional, la búsqueda de títulos y reconocimientos, los prejuicios existentes y la sesgada visión directiva, son obstáculos que imposibilitan alcanzar los objetivos señalados.

Para remediar esta situación partimos de la conceptualización de que una adecuada política de personal es aquella en la que prima el interés por las personas, teniendo como objetivo lograr su rápida y efectiva adaptación y conseguir su identificación con la empresa, lo que redundará en la consolidación de una imagen corporativa óptima. Es debido a esto que los programas de socialización no sólo buscan compenetrar a las personas con sus funciones y con las metas institucionales, sino que a la vez intentan contribuir a la consolidación de un clima en el que las relaciones humanas florezcan positivamente.

La propuesta que aquí se ha expuesto consiste en dar la responsabilidad de la ejecución de los programas de socialización al área de comunicaciones, es decir, que el comunicador social sea el encargado de realizar una labor coordinada con los distintos departamentos involucrados en el proceso de socialización. Para ello, las actividades a realizarse deberán comprender: a) la investigación: en la que se determinan las necesidades del público en materia inductiva, las que se detectan mediante la aplicación y análisis de encuestas y entrevistas en profundidad; b) la planificación: etapa posterior al diagnóstico en la que se programan las actividades informativas, su duración, fechas, horarios, lugares, expositores participantes, temas a tratar, materiales requeridos $\mathrm{y}$ otros puntos; c) la ejecución: consiste en la puesta en marcha de los planes establecidos y programados, promoviendo la adquisición de conocimientos y el desarrollo de las habilidades de los trabajadores, y d) el control: consiste en evaluar los resultados obtenidos. La unidad encargada de todas estas responsabilidades debería contar con suficientes recursos humanos y materiales.

También resulta primordial establecer y mantener relaciones de confianza con el personal, informándoles sobre los asuntos de su interés. Nunca se destacará lo suficiente el valor de una comunicación plena, en la que se minimizan las condiciones distorsionadoras y existe un intercambio constante de mensajes sin caer en la saturación. Coincidimos con Lougovoy cuando dice que: 
"La experiencia muestra que la empresa donde la información es la mejor es la más eficaz, y es porque el gerente moderno entiende que las politicas, objetivos, métodos, medios y el control de los resultados sean conocidos por todos"6.

Esta eficiencia se ha comprobado en aquellas entidades en las que los trabajadores conocen sus metas, planes y proyecciones, posibilitando que su rol como público interno sea activo y participativo.

\section{FASES DEL PROGRAMA DE SOCIALIZACIÓN}

Una primera etapa contempla la adaptación al ambiente institucional. El comunicador social es el responsable de preparar la sesión de bienvenida, de entregar información referente a la corporación (historia, organigrama, relación de bienes y servicios producidos, niveles de competencia, posición en el sistema económico y comercial, etc.), así como de resolver las interrogantes que se planteen. Sucesivamente se procede a la presentación de los ingresantes a sus superiores jerárquicos y al grupo de trabajo. Adicionalmente, se debe producir y realizar todo tipo de material gráfico y audiovisual (folletos, boletines, revista y video institucional, vitrinas, etc.) que facilite la ampliación y refuerzo de los temas tratados. Esto sirve, además, para evaluar el nivel de comprensión de los participantes, utilizando pruebas en las que se verifiquen los conocimientos adquiridos y se les motive para recoger sus apreciaciones y sugerencias respecto del programa.

La segunda fase comprende el acoplamiento del nuevo trabajador al ámbito laboral, aunque es verdad que aquí la responsabilidad mayor recae en el jefe inmediato. Toca a éste exponer las nociones detalladas acerca de las tareas a realizarse, normas, premios, sanciones, relación con otros departamentos y otros detalles específicos del lugar y puesto de trabajo. Debe también presentar a los compañeros con quienes se tendrá relación frecuente. Debe exponer el Manual de funciones y luego de algunas semanas aplicar la entrevista de ajuste.

\section{DURACIÓN DE LOS PROGRAMAS DE SOCIALIZACIÓN}

Éste varía de acuerdo con el tamaño de la empresa y sus características. Se sugiere un período que vaya entre un dia y una semana. En este proceso no se debe pasar por alto
6. Lougovoy,

Constantin.

Evolución y

perspectivas de las relaciones públicas europeas

contemporáneas.

Barcelona: Editorial

Hispano Europea.

Colección Estudios

de la Empresa,

1972. 
la función educativa de los programas, en cuanto son parte de una filosofia educacional que impulsa los planes de desarrollo organizacional. Desafortunadamente existen empresas que no entienden la seriedad y consistencia de este proceso, considerándolo innecesario o como un gasto inútil, sin comprender que se trata de una inversión que toda entidad puede y debe efectuar, en razón de que redituará beneficios concretos en diferentes plazos.

El proceso de socialización realizado en ciertos sistemas organizacionales recibe el nombre de inducción. Algunas de sus características principales son: a) actuar como un instrumento de capacitación; b) tener como público objetivo al personal de reciente incorporación; c) abarcar todos los puestos y niveles existentes; d) ser reflejo de una investigación exhaustiva de las particularidades de la entidad, del grupo y de las capacidades individuales; e) programarse en función de las necesidades de los participantes, posibilidades de la empresa y objetivos diseñados; f) ser flexible y adaptarse a los cambios; g) generar coordinaciones permanentes entre diversas áreas; h) contribuir en los niveles de identificación del personal y en la formación de una imagen óptima; i) necesitar un apoyo real de la Dirección General; j) ser el reflejo de una política organizacional esencialmente humanista, y k) coadyuvar al desarrollo de la institución.

A título de resumen presentamos una propuesta propia, tomada textualmente, que aún consideramos válida, acerca de la estructura que debe tener un programa inductivo y de socialización.

"El primer día de trabajo es, a no dudarlo, una experiencia que perdurará por siempre en la memoria del individuo. Este es un momento importante y definitivo en el logro de la identificación y efectiva incorporación del personal ingresante. Como sabemos, las personas que inician sus actividades por primera vez en una esfera organizacional exacerban sus necesidades de seguridad y aceptación. Si éstas no son satisfechas emergerán sentimientos de frustración que en el futuro pueden derivar en serios conflictos. La inducción, en esencia, considera estos aspectos y en líneas generales abarca: 1. La bienvenida. Es esencial brindar un recibimiento cordial a los empleados de reciente ingreso. De este modo la empresa demuestra su preocupación e interés. 2. Información general sobre la empresa, en la que se indica:

a) La historia de la organización.

b) Los bienes y servicios producidos.

c) El organigrama.

d) Las líneas de producción. 
e) Competencia existente.

f) Posición financiera.

g) Aspectos diversos que contribuyen al establecimiento de una imagen favorable.

3. Información acerca de las políticas de personal y la descripción detallada del puesto.

Comprende:

a) Los derechos del trabajador.

b) Los deberes del trabajador.

c) La descripción específica del cargo.

4. Otras comunicaciones:

Publicaciones de la empresa, eventos, programas de proyección familiar, entre otros.

Cabe indicar que la extensión y seriedad con que se aplique el plan de inducción implicará una relación directa entre el tipo específico de entidad a tratar, los recursos, las condiciones coyunturales y las politicas de dirección existentes.

A continuación detallamos las especificaciones del programa inductivo:

a) Se dirige a todo el personal ingresante.

b) Su duración dependerá de las características del puesto. Este aspecto será establecido de acuerdo a las necesidades de la organización.

c) En su desarrollo intervienen diferentes áreas como:

- Personal: Departamento que se encarga de brindar la información introductoria en la que se señalan entre otros puntos la fecha de incorporación, lugar de trabajo, horario, documentación a entregar, categoría laboral y retribución económica.

- Comunicaciones y/o Relaciones Públicas: Elabora el programa y se encarga de su difusión, produciendo los videos, folletos y material requerido por los instructores. Además organiza la bienvenida y presentación del personal.

- Seguridad Industrial: Se responsabiliza por el aprendizaje de las medidas de seguridad y dispositivos conexos.

- Servicio Social: Instruye sobre los beneficios asistenciales como el de seguridad social, de licencias por enfermedad, jubilación y otros.

- Sección que brinda información específica sobre el puesto de trabajo: esta enseñanza es impartida por personal especializado.

d) Finalizada la etapa de instrucción se sugiere aplicar una prueba a los ingresantes, a fin de verificar su grado de comprensión de los conocimientos impartidos, incluyéndose preguntas generales sobre la institución y el puesto laboral. Igualmente se aplicará un cuestionario a los ingresantes para evaluar el programa de inducción que se les ha aplicado. Las observaciones obtenidas servirán para realimentar y perfeccionar el proceso seguido. Asimismo, después de 10 o 12 semanas puede realizarse una entrevista de ajuste con el fin de conocer si los procesos de selección y socialización aplicados han sido adecuados, de forma tal que se puedan impartir correctivos en caso de existir alguna tergiversación"7

7. Huerta Álvarez, Rocío."La comunicación organizacional, los programas de inducción del personal y su importancia en la dinámica de la organización". Tesis presentada para optar el título de licenciada en ciencias de la comunicación. Tomo II capítulo 4. Lima:

Universidad de Lima, 1991. 
En relación con el contenido del programa sugerimos considerar los siguientes puntos:

Título

: Inducción de personal.

Elaborado por : (Nombre del encargado).

Aprobado por : (Responsable del área).

Objetivo

: Lograr eficiente adecuación y socialización del personal.

Tipo de sesión : Conferencia, seminario, etc.

Participantes : Personal de reciente ingreso.

Moderador : Ejecuta la sesión.

Características

del local : Distribución física del ambiente.

Material a emplear: Folletos, boletines y otros materiales.

Actividades a realizar:

a) Reunión con el personal nuevo durante el primer día de trabajo. Puede utilizarse la técnica de mesa redonda.

b) Información y explicación amplia del Manual de bienvenida. Este material considera:

- La historia de la organización.

- Sus objetivos.

- Horarios, dias de pago, tarjetero, reloj marcador, vacaciones, etc.

- Artículos producidos y/o servicios prestados.

- El organigrama de la institución identificando los puestos que se están completando.

- Políticas de personal.

- Prestaciones.

- La ubicación de los servicios como el comedor, enfermería, sanitarios, lavabos, etc., incluyendo un plano de las instalaciones. También se señalará el lugar en el que se colocarán las vitrinas, paneles y franelógrafos.

- El reglamento interno de trabajo.

- Cualquier otra información que puede ser de interés de las personas (por ejemplo, el procedimiento a seguir en caso de accidentes).

c) Exposición detallada del Manual de funciones y descripción de puestos.

Posteriormente se realizarán las siguientes actividades:

- Evaluación: Se aplica un cuestionario con el que se obtendrán datos sobre el nivel de comprensión del público objetivo.

- Visita guiada por las instalaciones: Propicia que los nuevos empleados se familiaricen con la distribución fisica del local. 
- Presentación ante los representantes de los trabajadores: Se trata de que entablen una relación con los delegados sindicales, si los hubiera, así como con aquellos empleados con quienes se mantendrá una estrecha relación.

- Entrega de información detallada por parte del superior jerárquico.

- Presentación del personal de reciente ingreso a los miembros del grupo de trabajo: De ser posible, se recomienda nombrar a uno de ellos para que asista a los nuevos trabajadores en los aspectos que necesite conocer.

- Impartir instrucciones de refuerzo: Se ampliarán los temas tratados en la sesión de bienvenida.

- Realizar la entrevista de ajuste: Es responsabilidad del jefe de Área.

Cabe indicar ciertos requisitos adicionales que se deben considerar al confeccionarse el programa de socialización:

a) Debe adecuarse a las necesidades del segmento al que se dirige. En este sentido, el ritmo de la enseñanza debe concordar con la capacidad de asimilación de las personas, tal como lo recomiendan los autores G. Strauss y L.R. Sayles: "Los programas de inducción tienen que estar encaminados a los problemas propios de los alumnos y tienen que estar adaptados en forma realista a sus necesidades"8.

b) Debe ser diseñado y elaborado en función de los objetivos propuestos, tomando en consideración los requerimientos y posibilidades de la entidad.

c) Ha de adaptarse a los cambios de politicas institucionales, así como a los generados por factores externos.

d) Se debe orientar a resolver los problemas existentes, estructurándose con criterios prácticos y realizables.

e) Debe comprender a todo el personal de la organización. No es suficiente establecer actividades diferentes según los planos jerárquicos. Es preciso, igualmente, que su realización se lleve a cabo por separado con el fin de evitar posibles conflictos y deslindar responsabilidades entre los departamentos involucrados.

f) Debe contener enseñanzas prácticas y concretas, evitando las generalizaciones y teorias.

g) Ha de contar para su realización con los medios requeridos de orden personal, material y económi-
8. Strauss, G. [y] L.R. Sayles. Los problemas humanos de la dirección. México: 1961, p. 637. 
co, de modo tal que el programa pueda aplicarse en el momento oportuno, es decir cuando las circunstancias y el clima sean más propicios.

h) Debe aplicarse de una manera continua, con tenacidad, paciencia y perseverancia, a pesar de las dificultades que se presenten sobre todo en las primeras fases.

i) Debe ofrecer cierta flexibilidad que le permita adaptarse a las circunstancias de cada momento sin perder de vista los fines perseguidos. Uno de los mayores peligros que amenaza el éxito del plan es la rigidez en su aplicación. Se precisa, en todo instante, de una gran capacidad de readaptación a la realidad cambiante.

j) Se debe establecer un control constante que implique la valoración de los resultados obtenidos. La evaluación tiene que aplicarse permanentemente.

Al llegar al final de este artículo, debemos destacar que las sugerencias expuestas con el propósito de tener éxito en la implementación del programa de socialización, se encaminan a evitar la obsolescencia de los datos e informaciones de la empresa, como los relativos al organigrama, a su nivel de participación en el mercado, al Manual de funciones, entre otros. Se trata de impedir las distorsiones que provoquen confusión e incertidumbre.

Por otro lado, se recomienda establecer un cronograma integral de todas las actividades a realizarse, pues éste constituiría un auxiliar importante para ejercer las actividades posteriores de control. Se sugiere, igualmente, verificar a través de un sistema de evaluación, que las aptitudes y capacidades del personal que aplica la inducción sean las requeridas, así como designar en cada departamento al personal que colaborará en el programa, que deben ser aquéllos que cuenten con la calificación y capacitación respectiva.

En la exposición hemos querido dilucidar algunos aspectos propios de los procesos de socialización en las empresas, así como la importancia y trascendencia de éstos respecto del público interno. Asimismo, hemos insistido en la necesidad de contar con el apoyo real de los mandos directivos, puesto que sin éste no se alcanzarán las metas propuestas.

Para concluir, sólo queda resaltar el impacto que ha de tener el programa de socialización en la constitución de una imagen corporativa integral, la cual es el resultado de una gestión planificadora que busca proyectar la filosofia empresarial que le es propia. Lograr la adecuada percepción de aquélla, por parte de los públicos con los que se establece 
interacciones, implica organizar actividades interdependientes e interdisciplinarias en las que resulta fundamental el concurso y la convicción de todos los involucrados.

Los programas de socialización, cuya característica hemos analizado en este artículo, representan sólo uno más de los arduos caminos a seguir para alcanzar los objetivos de constituir una imagen empresarial integral. Queda en manos de los responsables de la dirección empresarial, tomar las decisiones finales que concurran mejor a la consecución de las metas propuestas. 
Bibliografía

Arias Galicia, Fernando

1979

"La motivación", en Rodil Urrego, F. y F. Mendoza Trejo (editores), Conceptos fundamentales sobre la organización. México: Editorial Trillas S.A., p. 113.

Huerta Álvarez, Rocío 1991

"La comunicación organizacional, los programas de inducción del personal y su importancia en la dinámica de la organización”. Tesis presentada para optar el título de licenciada en ciencias de la comunicación. Tomo II, capítulo 4. Lima: Universidad de Lima, Facultad de Ciencias de la Comunicación.

Lougovoy, Constantin 1972

Evolución y perspectivas de las relaciones públicas europeas contemporáneas. Barcelona: Editorial Hispano Europea. Colección Estudio de la Empresa, p. 18.

Myers, Gail E. [y] Michelle Tolela Myers 1981

Administración mediante la comunicación: Un enfoque organizacional. México: McGraw Hill, p. 23.

Paredes Guzmán, Carlos 1991

Concepto de sistemas. Lima: Universidad de Lima, Facultad de Ciencias de la Comunicación.

Strauss, G. [y] L.R. Sayles 1961

Los problemas humanos de la dirección. México, p. 637.

Werther, William B. [y] Keith Davis 1981

Dirección de personal y recursos humanos. México: McGraw Hill, p. 153. 